\title{
ESTABILIDAD DE RENDIMIENTO DE LA LÍNEA DE FRIJOL NEGRO DOR-500 EN EL TRÓPICO HÚMEDO DE MÉXICO
}

\author{
Ernesto López S.1, Jorge A. Acosta G. ${ }^{2}$, Octavio Cano R. ${ }^{1}$, Guillermo Fraire V. ${ }^{1}$, \\ Javier Cumpian G. ${ }^{1}$, Enrique Noe Becerra L. ${ }^{1}$, Bernardo Villar S. ${ }^{3}$, Francisco J. Ugalde ${ }^{1}$
}

\section{RESUMEN}

Estabilidad de rendimiento de la línea de frijol negro DOR-500 en el trópico húmedo de México. El presente estudio se llevó a cabo durante los años 1994 a 1997. Se evaluó la adaptación de la línea DOR-500 en ocho experimentos establecidos en el trópico húmedo de México, cinco en el Estado de Veracruz, dos en Chiapas y uno en Guerrero. La línea DOR-500 resultó sobresaliente por su rendimiento, amplia adaptación y estabilidad (1063 kg/ha,bi=1.0,S2di=0). Además, DOR-500 se estableció en parcelas comerciales en diez localidades del Sureste de México (climas tropical), en éstas DOR-500 se comparó con testigos regionales y locales, y obtuvo un rendimiento medio de $1327 \mathrm{~kg} / \mathrm{ha}$. La línea DOR-500 fue tolerante a las enfermedades mosaico dorado, (BGMV) mancha angular (Phaseoisariopsis griseola) y roya (Uromyces appendiculatus). La línea Dor-500 está en trámite para su registro, como nueva variedad para las regiones productoras del trópico húmedo de México.

\begin{abstract}
Yield stability of the black bean DOR-500 line in the humid tropic from Mexico. The present study was carried out during 1994 to 1997. Adaptation of the DOR-500 line was evaluated in eight experiments established in the humid tropic of Mexico, five in the state of Veracruz, two in Chiapas and one in Guerrero. The line DOR-500 was excellent according of its yield, wide adaptation and stability (1063/ $\mathrm{kg} / \mathrm{ha}$, bi=1.0, S2di=0). DOR-500 line was established in commercial plots in ten locations of the Mexican Southeast (tropical climate) comparing this line to the regional and local control treatments; it was obtained a yield of 1327 $\mathrm{kg} / \mathrm{ha}$. The DOR-500 line was tolerant to the bean golden mosaic virus (BGMV), Leaf spot (Phaseoisariopsis griseola), and rust (Uromyces appendiculatus). The Dor-500 line is being registered as a new variety for the production regions of Mexico.
\end{abstract}

\section{INTRODUCCIÓN}

Las variedades mejoradas de frijol se pueden obtener por los método de introducción, selección e hibridación. El método de introducción consiste en introducir a una localidad o región germoplasma que ha sido desarrollado en otras regiones (Miranda, 1996). La evaluación sistemática de materiales importados pueden dar los mismos beneficios que los logrados con los métodos de mejoramiento convencionales CIAT (1986). El programa de frijol de Veracruz, México, introdujo en 1980 la línea D-145, del Proyecto BGMV CIAT-ICTA, misma que resultó mas tolerante al BGMV que las variedades locales (Jamapa Y Negro Veracruz). D-145 fue liberada en 1982, como Negro Huasteco-81 (Yoshii Y Rodríguez et al., (1982). Este mismo programa en los 90's generó las líneas E-46 y E-47, producto de la cruza D-149 X 1397, líneas introducidas de el proyecto arriba mencionado. En 1991 y 1992 se liberaron las variedades Negro Cotaxtla-91 y Negro INIFAP, derivadas de la línea E46 Y E47 respectivamente (López et al, 1993 y López et al, 1994). En ésta década se introdujo la línea DOR-390, la cual mostró mayor resistencia al BGMV, que las variedades antes liberadas en el Sureste de México. Además, mostró tolerancia a otras enfermedades (complejo virótico, BMMV, BCMV y BSMV, mancha angular, roya y antracnosis). La línea DOR-390 fue liberada en 1993, como Negro Tacaná (López et al., 1994).

La introducción de poblaciones segregantes así como líneas del proyecto PROFRIJOL-CIAT-COSUDE, ha permitido que el programa regional de frijol del tró-

\footnotetext{
1 Investigadores del Programa Frijol CECOT-CIRGOC-INIFAP. Apartado Postal No. 429 Veracruz, Ver. México.

2 Líder Nacional del Programa Frijol CEVAMEX-CIR-CEN-INIFAP. Apartado Postal No. 10, Chapingo, Méx. México.

3 Investigador del Programa Frijol. CECOT-CIRPAS-INIFAP. Apdo. Postal No. 1. Ocozocuautla, Chis, México.
} 
pico de México, identifique genotipos sobresalientes los cuales se pueden liberar como nuevas variedades o incluirse en los programas locales de hibridación.

El objetivo del presente trabajo fue evaluar la adaptación y rendimiento de la línea DOR-500, introducida a través del proyecto arriba mencionado, en el Trópico Húmedo de México.

\section{MATERIALES Y MÉTODOS}

\section{Evaluación de rendimiento de la línea de frijol DOR-500}

Durante el periodo 1994 a 1997, se condujeron ocho experimentos uniformes de rendimiento, bajo condiciones de humedad residual y temporal; cinco en el estado de Veracruz, dos en Chiapas y uno en Guerrero. Las principales características de los sitios de prueba se presentan en el Cuadro 1. En los ensayos se incluyó la línea DOR-500 y los testigos Negro Jamapa y Negro Tacaná, que son de las principales variedades recomendadas para la zona tropical húmeda de México. En todos los ensayos se utilizó un diseño de Bloques completos al Azar con tres repeticiones y parcelas de tres surcos de cinco milímetros de longitud.

El rendimiento de grano se calculo en $\mathrm{kg} / \mathrm{ha}^{-1}$ al $14 \%$ de humedad. Los datos se sometieron a un análisis de varianza individual y después se realizó un análisis combinado de los ocho experimentos, para comparar las medias de tratamiento por la prueba Tukey a un nível de significación del 0,05\%. También se realizó un análisis de regresión con los ocho experimentos por el método de Eberhart y Russell (1966) para después hacer la calificación de variedades con base al coeficiente de regresión y las desviaron de la regresión (Carballo y Márquez, 1970).

\section{Evaluación de parcelas de validación}

Se evaluó la línea DOR-500 en diez parcelas de validación en el Sureste de México durante el ciclo agríco- la de 1998-99, comparándose con variedades locales y líneas experimentales. La superficie por lote de prueba fue variable en cada sitio y el rendimiento se calculo en $\mathrm{kg} / \mathrm{ha}$ en base al área total sembrada. Estas parcelas se establecieron en terrenos de agricultores cooperantes.

\section{Evaluacion a las enfermedades.}

Durante la conducción del ensayo uniforme de 1994 a 1997, se calificó la reacción de DOR-500 a la incidencia del mosaico dorado y mancha angular en el centro y sur de Chiapas, según la escala 1-9, propuesta por el Centro Internacional de Agricultura tropical (CIAT), cuyos valores son: $1-2=$ resistente, $3-4=$ tolerantes, 5 y $6=$ medianamente tolerantes, 7 y $8=$ medianamente susceptibles y $9=$ susceptible. También se calificó la reacción a roya, con una escala en 1-5 (tomada de CIAT, 1987), según la cual 1 = inmune; 2 = resistente; 3 = moderadamente resistente; $4=$ moderadamente susceptible y $5=$ susceptible.

\section{RESULTADOS Y DISCUSIÓN}

\section{Evaluación de rendimiento}

Los resultados de los experimentos individuales y en conjunto, de 16 variedades de frijol, evaluadas en ocho ambientes del trópico húmedo de México, se presentan en el Cuadro 2. En este se advierte que en la mayoría de los sitios existieron diferencias altamente significativas en los análisis individuales para variedades y en el conjunto, para variedades, localidades y variedad por localidad. Los genotipos que mostraron los mejores rendimientos en el análisis de conjunto fueron: II-307-CB-5E-OE-M-M, II68F60C-29,1713,1467 Y DOR-500, que resultaron estadísticamente iguales entre sí y con las variedades comerciales Negro Cotaxtla-91 y Negro Tacaná, pero diferentes a Negro Jamapa. Las localidades en que se obtuvieron los mejores rendimientos fueron: Zitlala, Guerrero y Tihuatlan, Ver., y las que mostraron los rendimientos más bajos fueron: Ocozocoautla 95-96 y 97-98 debido a un severo ataque de mosaico dora-

Cuadro 1. Localización geográfico, temperatura y precipitación de las localidades donde se realizaron los ensayos.

\begin{tabular}{lccccc}
\hline Localidad & Latitud & Longitud & $\begin{array}{c}\text { Altitud } \\
(\mathbf{m s n m})\end{array}$ & $\begin{array}{c}\text { Temperatura } \\
\text { media anual }\left({ }^{\circ} \mathbf{C}\right)\end{array}$ & $\begin{array}{c}\text { Precipitación } \\
(\mathbf{m m})\end{array}$ \\
\hline CEC-Veracuz-Centro & $19^{\circ} 12^{\prime}$, & $96^{\circ} 81^{\prime}$ & 16 & 25.2 & 1668 \\
CEPAP-Veracuz-Sur & $18^{\circ} 06^{\prime}$ & $95^{\circ} 53^{\prime}$ & 25 & 25.0 & 1762 \\
CEPAPAN-Veracuz-Norte & $20^{\circ} 57^{\prime}$ & $97^{\circ} 24^{\prime}$ & 14 & 24.2 & 1351 \\
CECCH-Chiapas-Centro & $16^{\circ} 46^{\prime}$ & $93^{\circ} 22^{\prime}$ & 846 & 23.6 & 898 \\
CEIGUA-Guerrero-Zitlala & $17^{\circ} 19^{\prime}$ & $98^{\circ} 58^{\prime}$ & 1420 & 20.0 & 1200 \\
\hline
\end{tabular}


Cuadro 2. Rendimiento expresado en $\mathrm{kg} / \mathrm{ha}$ al $14 \%$ de humedad de 16 variedades de frijol del eur 1994-1997, sembrados en ocho ambientes del trópico humedo de Mexico.. México, 1997.

\begin{tabular}{|c|c|c|c|c|c|c|c|c|c|c|}
\hline \multirow[t]{2}{*}{$\begin{array}{l}\text { Linea } \\
\text { Y/O } \\
\text { Variedad }\end{array}$} & \multicolumn{5}{|c|}{ Veracruz } & \multicolumn{2}{|c|}{ Chiapas } & \multirow{2}{*}{$\begin{array}{c}\begin{array}{c}\text { Guerrero } \\
\text { Promen } \\
\text { kg/ha }\end{array} \\
7 \text { ZITA8 }\end{array}$} & \multirow[t]{2}{*}{$\begin{array}{l}\text { Rend. } \\
\text { Tukey } \\
(\mathbf{0 . 0 5 )}\end{array}$} & \multirow[t]{2}{*}{ Prueba } \\
\hline & ISLA1 & ISLA2 & ISLA3 & TIH4 & CECOT5 & OCOZO6 & OCOZO7 & & & \\
\hline II-307-CB-5E-M & 1091 & 1022 & 1130 & 1902 & 863 & 1000 & 733 & 2109 & 1231 & $\mathrm{a}$ \\
\hline IIFGOC-29-M-M & 824 & 1062 & 1041 & 1703 & 787 & 912 & 525 & 1880 & 1092 & $a b$ \\
\hline 1713 & 1039 & 1003 & 927 & 1473 & 814 & 716 & 524 & 2052 & 1068 & $a b$ \\
\hline 1467 & 956 & 1073 & 1120 & 1461 & 979 & 409 & 560 & 1971 & 1066 & $a b$ \\
\hline DOR-500 & 1223 & 1029 & 1175 & 1296 & 759 & 1280 & 554 & 1191 & 1063 & $a b$ \\
\hline 1670 & 722 & 946 & 894 & 1181 & 626 & 1008 & 544 & 1924 & 980 & $\mathrm{bc}$ \\
\hline SM-98 & 1005 & 909 & 1030 & 1592 & 804 & 663 & 535 & 1295 & 979 & $\mathrm{bc}$ \\
\hline 1671 & 1007 & 975 & 875 & 1466 & 509 & 678 & 561 & 1674 & 968 & $\mathrm{bc}$ \\
\hline II-283-CB-5E-M-M & 1091 & 873 & 1223 & 1566 & 1119 & 614 & 568 & 633 & 961 & $\mathrm{bc}$ \\
\hline 1684 & 763 & 906 & 953 & 1370 & 757 & 935 & 558 & 1300 & 943 & $\mathrm{bc}$ \\
\hline 1708 & 1020 & 934 & 1030 & 1295 & 644 & 855 & 680 & 1048 & 938 & $\mathrm{bc}$ \\
\hline N-COTAXTLA-91 & 765 & 878 & 1022 & 1189 & 683 & 679 & 597 & 1657 & 933 & $\mathrm{bc}$ \\
\hline N.TACANA & 1109 & 1051 & 1194 & 713 & 1070 & 592 & 472 & 1135 & 917 & bcd \\
\hline SM-52 & 885 & 585 & 1127 & 1173 & 916 & 528 & 527 & 1301 & 880 & $\mathrm{~cd}$ \\
\hline DOR-446 & 845 & 915 & 873 & 1393 & 738 & 907 & 565 & 752 & 873 & $\mathrm{~cd}$ \\
\hline JAMAPA & 640 & 893 & 954 & 1087 & 827 & 256 & 584 & 749 & 749 & $\mathrm{~d}$ \\
\hline PROMEDIO & 937 & 941 & 1035 & 1366 & 806 & 752 & 568 & 1417 & 978 & \\
\hline C.V.\% & 20.9 & 14.1 & 16.1 & 23.0 & 17.0 & 20.0 & 15.7 & 22.8 & 18.21 & \\
\hline ANDEVA & $* *$ & $* *$ & N.S & $*$ & $* *$ & $* *$ & $* *$ & $* *$ & $* *$ & \\
\hline LOC & & & & & & & & & $* *$ & \\
\hline LXT & & & & & & & & & $* *$ & \\
\hline
\end{tabular}

1,2 y 3 Campo Experimental Papaloapan, En Isla,Veracruz; 4 Tihuatlan, En Veracruz, 5=Campo Experimental Cotaxtla En Veracruz; 6 Y 7 = Ocozocoautla, En Chiapas; 8= Zitla, En Guerrero.

do y falta de humedad en las etapas reproductivas del cultivo, respectivamente (Cuadro 3).

Los rendimientos promedio de ocho ambientes, los valores de los parámetros $\mathrm{Bi}$ y $\mathrm{S}^{2} \mathrm{di}$, y la calificación de cada uno de los genotipos con base en el esquema propuesto por Carballo y Márquez (1970), se presentan en el Cuadro 4. De los 16 genotipos evaluados para rendimiento en los ocho ambientes, 10 fueron estables $\left(\mathrm{Bi}=1 ; \mathrm{S}^{2} \mathrm{di}=0\right)$; dentro de esta categoría se encontraron las variedades Negro Cotaxtla-91 y Jamapa, además de ocho líneas experimentales, incluyendo a DOR-500. Adicionalmente, tres genotipos $\left(\mathrm{Bi}=0 \mathrm{~S}^{2} \mathrm{idi}>0\right)$ mostraron buena respuesta en todos los ambientes pero inconsistentes. Las líneas experimentales II-307-CB-5E-0EM-M y II68 F60C-29 con (bi $>1, S^{2} \mathrm{di}=0$ ) respondieron mejor en ambientes buenos y fueron consistentes. Por último la línea 1708 , se adaptó mejor en ambientes desfavorables y fue consistente (bi<1, $\mathrm{S}^{2} \mathrm{di}=0$ ). Los resultados anteriores difieren con los reportados por López

Cuadro 3. Promedio de rendimiento de 16 líneas y variedades de frijol en ocho ambientes del trópico húmedo de México. 1994-1997.

\begin{tabular}{lcccc}
\hline Localidad & Ciclo Agrícola y año & Condición & Rend. kg/ha & Tukey (0,05) \\
\hline Zitlala, Guerrero & PV-1997-1998 & T & 1417 & A \\
Tihuatlán,Veracruz & OI-1994-1995 & HR & 1366 & A \\
Isla,Veracruz & OI-1996-1997 & HR & 1035 & B \\
Isla,Veracruz & OI-1995-1996 & HR & 941 & B \\
Isla,Veracruz & OI-1994-1995 & HR & 937 & B \\
Cecot,Veracruz & OI-1996-1997 & HR & 806 & C \\
Ocozocoautla, Chiapas & OI-1995-1996 & HR & 752 & C \\
Ocozocoautla,Chis. & OI-1997-1998 & HR & 568 & D \\
\hline
\end{tabular}

OI = Otoño-Invierno PV = Primavera-Verano $\mathrm{HR}=$ Húmedo el residual $\mathrm{T}=$ Temporal 
Cuadro 4. Rendimiento promedio y parámetros de estabilidad de 16 líneas y variedades de frijol en ocho ambientes del trópico húmedo de México 1994-1997-CECOT-CIRGOC-INIFAP.

\begin{tabular}{|c|c|c|c|c|}
\hline Línea y/o Variedad & $\begin{array}{l}\text { Rend. } \\
\text { (kg/ha) }\end{array}$ & $\begin{array}{r}\text { Coeficiente de } \\
\text { (bi)Regresión }\end{array}$ & $\begin{array}{c}\text { Desviasion de } \\
\text { Regresión S2 di }\end{array}$ & $\begin{array}{c}\text { Calificacion de } \\
\text { acuerdo a Carballo } \\
\text { y Marquez }\end{array}$ \\
\hline II-307-CB-5E-OE-M-M-M & 1231 & 1,63 & $-6,994.95$ & d \\
\hline II-68-FGOC-29-M-M & 1092 & 1,53 & $-13,640.24$ & $\mathrm{~d}$ \\
\hline 1713 & 1068 & 1,56 & 568.68 & a \\
\hline 1467 & 1066 & 1,55 & $8,974.28$ & a \\
\hline DOR-500 & 1063 & 0,60 & $16,973.73$ & $\mathrm{a}$ \\
\hline 1670 & 980 & 1,22 & $39,295.73$ & $\mathrm{~b}$ \\
\hline SM-98 & 979 & 1,1 & $-19,491.13$ & a \\
\hline 1671 & 968 & 1,35 & $-10,343.26$ & a \\
\hline II-283-CB-5E-M-M-M & 961 & 0,53 & $84,885.57$ & $\mathrm{~b}$ \\
\hline 1684 & 943 & 0,87 & $-20,282.62$ & a \\
\hline 1708 & 938 & 0,61 & $-16,169.84$ & $\mathrm{c}$ \\
\hline NC-91 & 933 & 1,12 & $-11,776.48$ & $\mathrm{a}$ \\
\hline N.TACANA & 917 & 0,42 & $42,332.92$ & $\mathrm{~b}$ \\
\hline SM-52 & 880 & 0,90 & $-3,238.12$ & $\mathrm{a}$ \\
\hline DOR-446 & 873 & 0,48 & $11,764.23$ & $\mathrm{a}$ \\
\hline JAMAPA & 749 & 0,51 & $19,383.17$ & $\mathrm{a}$ \\
\hline PROMEDIO & & 978 & & \\
\hline \multirow[t]{5}{*}{ C.V. $\%$} & & 18,21 & & \\
\hline & & ANDEVA & & \\
\hline & & AMBIENTE & $* *$ & \\
\hline & & VARIEDAD & $* *$ & \\
\hline & & VXA & $* *$ & \\
\hline
\end{tabular}

\footnotetext{
a Estable

b Buena respuesta en todos los ambientes, inconsistente

c Responde mejor en ambiente desfavorable, consistente

d Responde mejor en buenos ambientes, consistente.
}

et al (1996), donde las líneas II-307-CB-5E-OE-M-M y II-68-FG0C-29, DOR-390 y Jamapa, mostraron diferente calificación a la de este trabajo, mientras los resultados reportada con las variedades comerciales Jamapa y Negro Cotaxtla-91 fueron similares.

\section{Evaluacion en parcelas comerciales de validación}

La evaluación de la línea DOR-500 en diez parcelas de validación establecidas en Veracruz y Chiapas, mostró un rendimiento medio de $1327 \mathrm{~kg} / \mathrm{ha}^{-1}$ (Cuadro 5). Producción similar a la obtenida por la mejor línea del presente estudio II-307-CB-5E-M-M, con 1300 kg/ha y muy superior a los rendimientos de las variedades locales, las cuales mostraron susceptibilidad a las enfermedades roya y mancha angular.

\section{Evaluación de enfermedades}

La enfermedad del virus de mosaico dorado se evaluó en el centro de Chiapas (Ocozocoautla), en los ciclos Otoño-Invierno 95-96 y 98-99, en este último ciclo se calificó también la reacción a la mancha angular . (Cuadro 6). En el Sur de Chiapas (Tuxtla Chico) se calificó el BGMV, en el ciclo Otoño-Invierno 92-93. La línea DOR-500 mostró tolerancia al BGMV y a la mancha angular en el centro y sur de Chiapas, mientras que Jamapa fue susceptible al BGMV, en el Sur de Chiapas. Confirmando lo reportado por Yoshii et al (1987); López et al (1996); López et al, (1993).

La reacción a la roya se calificó en el centro de Veracruz, en los ciclos Primavera-Verano 94-94 y 97-97 y en el norte de Veracruz, en el ciclo Primavera-Verano 93-93. Los resultados indicaron que DOR500 y Negro Tacaná fueron resistentes, en cambio la variedad Jamapa resulto susceptible a la roya (Cuadro 7). Los resultados fueron similares a los reportados por López et al (1993) López et al, 1994 López et al (1993). 


\section{CONCLUSIONES}

Se identificaron diez genotipos estables en rendimiento a través de las localidades de prueba.

Se observaron diferencias significativas en rendimiento entre genotipos a través de localidades y para la interacción localidad por genotipo.
Las localidades, de mayor potencial de rendimiento fueron: Zitlala en Guerrero y Tihuatlan, en Veracruz con un rendimiento media aproximada a $1400 \mathrm{~kg} / \mathrm{ha}$.

Los genotipos que mostraron los mayores rendimientos fueron: II 307-CB-5E-OE-M-M, II68 F60C-29, 1713,1467 y DOR-500, con rendimientos hasta $1231 \mathrm{~kg}$ /ha. El menor rendimiento promedio se observó con el testigo Jamapa con $749 \mathrm{~kg} / \mathrm{ha}$.

Cuadro 5. Rendimiento promedio parcela de validación de las líneas dor-500 y ii-307, en el sureste de México. CIRGOCINIFAP. 1997.

\begin{tabular}{lcccc}
\hline Localidad & Ciclo y año & \multicolumn{3}{c}{ Rendimiento $\mathbf{k g} / \mathbf{h a}$} \\
\cline { 3 - 5 } & & DOR-500 & II-307 & Variedades \\
\hline Veracruz,Ver. & OI-1998-1999 & 1917 & 1597 & 762 CRIOLLO \\
Veracruz,Ver. & OI-1998-1999 & 1252 & 838 & 580 JAMAPA \\
Jamapa,Ver. & OI-1998-1999 & 1911 & 2105 & 1000 BOLITA \\
M.de Bravo, Ver. & OI-1997-1998 & 2000 & 1661 & ------ \\
M.de Bravo,Ver. & OI-1998-1999 & 996 & 864 & 600 JAMAPA \\
Ocozocoautla, Chis. & OI-1998-1999 & 760 & 673 & 762 N.INIFAP \\
Ixtacuaco,Ver. & OI-1998-1999 & 1353 & 1539 & 1171 MICHIGAN \\
Isla,Ver. & OI-1998-1999 & 1081 & 959 & 620 JAMAPA \\
San Andrés Tuxtla,Ver. & OI-1998-1999 & 1426 & 1756 & 600 C.REGIONAL \\
Isla,Ver. & OI-1998-1999 & 572 & 1010 & 931 N.TACANA \\
Total & & 1327 & 1300 & \\
\hline
\end{tabular}

Cuadro 6. Reacción al virus del mosaico dorado y mancha angular en línea. DOR-500 de frijol en Chiapas en tres ciclos DE SIEMBRA ${ }^{1 /}$. MÉXICO, 1997.

\begin{tabular}{lcccc}
\hline Variedad & \multicolumn{5}{c}{ Localidades } \\
\cline { 2 - 5 } & $\begin{array}{c}\text { Tuxtla Chico } \\
\text { Bgmv }\end{array}$ & $\begin{array}{c}\text { Ocozocoautla } \\
\text { Bgmv }\end{array}$ & $\begin{array}{c}\text { Ocozocoautla } \\
\text { Bgmv }\end{array}$ & $\begin{array}{c}\text { Ocozocoautla }_{\mathbf{4}} \\
\text { M.Angular }\end{array}$ \\
\hline DOR-500 & 2 & 1 & 3 & 3 \\
JAMAPA & 8 & - & - & - \\
N.INIFAP & 3 & 2 & - & - \\
N.HUASTECO-81 & 5 & - & - & 4 \\
N.TACANA & 2 & - & 5 & - \\
ICTA OSTUA & - & - & & \\
\hline
\end{tabular}

1/ Escala de calificación de BGMV y mancha angular: 1-2 = Resistente; 3-4 = tolerante; 5-6= medianamente tolerante; $7-8=$ medianamente susceptible, $9=$ susceptible.

2/ Ciclo de siembra Otoño-Invierno de 1992-93.

3/ Ciclo de siembra Otoño-Invierno de 1995-96.

4/ Ciclo de siembra Otoño-Invierno de 1998-99. 
Cuadro 7. Reacción a roya de las líneas dor-500 y variedades de frijol en el centro de Veracruz, México, 1997.

\begin{tabular}{lccc}
\hline $\begin{array}{l}\text { Localidades } \\
\text { Variedad }\end{array}$ & Tihuatlan $^{\mathbf{1}}$ & I.de la llave $^{\mathbf{2}}$ & CECOT $^{3}$ \\
\hline DOR-500 & 3,0 & 2,0 & 2,0 \\
Negro Tacana & 3,0 & 2,0 & 2,0 \\
Negro Jamapa & 5,0 & 5,0 & 3,5 \\
\hline
\end{tabular}

Ciclos de Primavera-Verano: 1/ P-V 93-93

$$
\begin{array}{lll}
\text { 2/ } & \mathrm{P}-\mathrm{V} & 94-94 \\
\text { 3/ } & \mathrm{P}-\mathrm{V} & 97-97
\end{array}
$$

Escala de calicación de roya: 1 = inmune, 2 = resistente, 3 = moderadamente resistente, $4=$ moderadamente susceptible 5 = susceptible.

\section{LITERATURA CITADA}

CARBALlO, C.A; MÁRQUEZ, S. 1970 Comparación de variedades de maíz del Bajío y la mesa central por un rendimiento y estabilidad. Agrociencia 5: 129-146

CENTRO INTERNACIONAL DE AGRICULTURA TROPICAL 1986. Mejoramiento de frijol por introducción y selección: guía de estudio para ser usada como complemento de la unidad audio tuturial sobre el mismo tema. Contenido científico: Oswaldo Vogset, Marceliano López G. Producción: Fernando Fernández O. Calí, Colombia 32 p. (serie 045B-08.03)

CENTRO INTERNACIONAL DE AGRICULTURA TROPICAL. 1987 sistema estándar para la evaluación de germoplasma de frijol. Art van Schoonchoven y Marcial A. Pastor Corrales (comps.) Calí, Colombia.

EBERHART, S.A.; RUSSELL,W. 1996. Stability parameters for comparing varieties. Crop. Sci. 6:36-40
LÓPEZ S.E., RODRÍGUEZ R.; BECERRA L.N.E.; CANO, R.O. 1993. Negro Cotaxtla-91, nueva variedad frijol para la zona tropical de Veracruz. Agron. Mesoam. 4: 81-85.

LÓPEZ S.E.; BECERRA L.N.E.; ACOSTA, G.A.J.; VILLAR, S.B. 1993. Variedades de frijol tolerantes al virus del mosaico dorado para el trópico de México. Agric.Téc.Méx.,Vol 19 núm. 2 99-109.

LÓPEZ,S.E.; VILLAR, S.B.; CANO, R.O. 1994. Rendimiento y adaptación del frijol al Negro E-44 en el Trópico Húmedo de México. Rev.fitotec.Mex.Vol.17:39-47.

LÓPEZ, S.E.; BECERRA, L.N.E.; CANO, R.O.; ORTEGA, Z.A.D.; ACOSTA G.A.J 1996. Adaptación y calidad tecnológica de la variedad de frijol Negro Tacaná. Agro. Mesoam. 7(1):26-34.

LÓPEZ, S.E.; CANO, R.O.; ACOSTA, G.A.; BECERRA, L.N.E.; CHAVEZ. C.F.; ORTEGA, D; VINAY, B.J. 1994. Negro Tacaná, nueva variedad de frijol para el trópico húmedo de México. Veracruz-México. SAGAR-INFAP-CIRGOC-CECOT. 18 p. (Folleto técnico num. 10)

MIRANDA, C.S. 1966. Mejoramiento del frijol en México. Folleto Misceláneo No.13. Instituto Nacional de Investigaciones Agrícolas, Secretaría de Agricultura y Recursos Hidráulicos. México 19p.

YOSHII, O.K.; RODRÍGUEZ, R.R.J.; NUÑEZ G.S.; ARRIZALES, M.N.; IBARRA, P.J.F.; PÉREZ, G.P. 1987. Rendimiento y adaptación de la línea D-145 tolerante al mosaico dorado del frijol en el Sureste de México. Fitotecnia, 9:57-63.

YOSHII, O.K.; RODRÍGUEZ; R.J.R. 1982. Negro Huasteco81. nueva variedad de frijol para el trópico húmedo de México-Veracruz México. Secretaría de Agricultura y Recursos Hidráulicos. Instituto Nacional de Investigaciones Agrícolas. Centro de Investigaciones del Golfo-Centro. Campo Experimental Cotaxtla. (Folleto técnico no. 1). 10 p. 